\title{
Bile Acids and the Potential Role in Primary Biliary Cirrhosis
}

\author{
Hang Yang Zhijun Duan \\ The Second Department of Gastroenterology, The First Affiliated Hospital of Dalian Medical University, Dalian, China
}

\section{Key Words}

Bile acids · Primary biliary cirrhosis · Gut microbiome .

Immune system $\cdot$ Hepatic stellate cell

\begin{abstract}
Background: Bile acids (BAs) play a potential role in regulating the whole-body metabolic homeostasis via the interaction with gut microbiome and the signal transduction as messengers, which establish a link between the primary biliary cirrhosis (PBC) and gut microbiome in many aspects, particularly with regard to the immune system of the body. PBC, as a chronic cholestatic liver disease characterised by the destruction of small intrahepatic bile ducts, causes fibrosis and potential cirrhosis without efficient therapies. Summary: Recent researches show BAs can induce the differentiation of hepatic stellate cells, suggesting that it may serve as a novel therapy to resist, even changeover the irreversible liver cirrhosis in PBC. Key Messages: In this review, we conclude and provide information on the possible mechanism of pleiotropic BAs in homeostasis of the gut microbiome and liver regeneration, and hope to broaden the therapy of PBC and promote the relevant drugs' development.
\end{abstract}

(c) 2016 S. Karger AG, Basel
(C) 2016 S. Karger AG, Basel

$0012-2823 / 16 / 0943-0145 \$ 39.50 / 0$

\section{Introduction}

As the main constituent of human bile, bile acids (BAs) are signalling molecules that regulate the self-formation, lipid-glucose-energy metabolism involved in the improvement of glucose tolerance and insulin sensitivity in metabolic disorders, activation of farnesoid X receptor (FXR), serum and lumen level and of fibroblast growth factor 19 (FGF19), and the induction of liver proliferation. Meanwhile, it stimulates sterol regulatory elementbinding protein-1c expression, which has a role in genes regulation of cholesterol biosynthesis, and in governing the expression of genes such as fatty acid synthase, acetylCoA carboxylase, and glycerol-3-phosphate acyltransferase, which are involved in lipogenesis [1] to protect against hyperlipidemia. And activation of FXR can also improve hyperlipidemia, glucose intolerance, and insulin sensitivity [2]. Furthermore, BAs also play an essential role in the permeability of mucosal barrier, have variable impacts on epithelia and innate immune cells, and have mutual effect with specific inflammatory factors to participate in the regulation of the intestinal and mucosal immune system and the composition and ecosystem of gut microbiome [3-5]. According to previous researches,

\section{KARGER}

E-Mail karger@karger.com www.karger.com/dig 
this review indicates that BAs are highly involved in the initiation, development, and progression of primary biliary cirrhosis (PBC) pathologies via the regulation homeostasis of gut microbiome and specific molecules, and serve a potent role in the induction of hepatic stellate cell (HSC), implicating liver regeneration through a potential replacement.

\section{Bile Acids}

The Metabolism of BAs

The 2 primary BAs in humans, cholic acid (CA) and chenodeoxycholic acid (CDCA) are synthesized by hepatocytes [6]. Cholesterol 7a-hydroxylase (CYP7a1), the rate-limiting enzyme, initiates the classic pathway for $\mathrm{BA}$ synthesis, and the sterol 12a-hydroxylase (CYP8b1) is required for CA synthesis. While CYP7a1 initiates the alternative pathways, part of BA undergo conjugation $(\mathrm{N}-\mathrm{acyl}$ amidation in the liver) with glycine or taurine. Thereafter, a fraction of the glycine/taurine conjugated primary BAs is deconjugated contributing to the production of ursodeoxycholic acid (UDCA) via epimerizing the 7a-hydroxy group of deconjugated CDCA by bacteria in the small bowel. Deconjugated CD, CDCA and UDCA diffuse passively through the intestinal epithelium while the conjugated BAs are actively transported by the apical sodium/ BA cotransporting polypeptide encoded by the apical sodium-dependent BA transporter gene expressing in the terminal ileum $[7,8]$, both of which return to the liver by the portal vein to complete the process of enterohepatic circulation. And the colonic microbial transconformation including deconjugation (removal of the amino acid side chain) and dehydroxylation (replacement of a hydroxyl group with a hydrogen), conversion of about $5 \%$ of conjugated BAs to secondary BA (deoxycholic acid (DCA) from CA and lithocholic acid (LCA) from CDCA), which are partly absorbed by colon and return to liver and partly lost in feces. DCA, LCA, UDCA, CA and CDCA are received by the liver after the reconjugation $[9,10]$.

\section{Receptors of BAs}

BAs, as signalling molecules and stimulators of several major signalling networks are known ligands for at least 5 members of the NR superfamily: FXR, the constitutive androstane receptor, the pregnane $\mathrm{X}$ receptor, the liver $\mathrm{X}$ receptor (LXR), the vitamin $D$ receptor, and secondary BAs activate G-protein-coupled receptors, including G protein-coupled bile acid receptor 1 (GPBAR1; also known as transmembrane G-protein-coupled bile acid receptor 5, TGR5), muscarinic receptors and sphingosine 1 receptor [11]. The key sensor and regulator of BA synthesis is the FXR highly expressed in liver and ileum [12], a nuclear receptor that binds to DNA response elements in hepatocytes such as SHP gene to inactivate both hepatic Hnf4a and Lrh-1 proposed to function as important transcription factors in the induction of CYP7al by transactivating CYP7a1 promoter activity resulting in a decrease in CYP7al expression and inhibition of BA synthesis. The activation of FXR in ileal enterocytes releases FGF19, which is mainly expressed in the ileum in response to luminal bile salt loading in the post-prandial state in humans, and secreted into portal blood to act as an endocrine factor that binds to hepatic FGF receptor 4/ Klotho- $\beta$ cell-surface receptor complex, and activates c-Jun $\mathrm{N}$-terminal kinase $1 / 2$ and extracellular signal regulated kinase $1 / 2$ to inhibit CYP7al and impede BA synthesis $[13,14]$, and also negatively regulates basolateral BA uptake by hepatocytes via the repression of NTCP/ solute carrier family 10 (sodium/BA cotransporter), member 1 (SCL10A1) and solute carrier organic anion transporter family, member $1 \mathrm{C} 1$ and -4 (OATP1/ SLCO1C1) [15]. Intestinal peroxisome proliferator-activated receptor-UDP-glucuronosyltransferases, the upstream of FXR-FGF19 signalling, also plays vital roles in control of BA homeostasis, activation of which reduces the intracellular levels of BAs in the intestine to compromise intestinal FXR-FGF19 signalling, leading to the continuous activation of liver CYP7a1, and thus increasing the de novo synthesis of BAs $[16,17]$.

And another main kind of receptor is GPBAR1, which is predominantly expressed in the ileum and colon; its activation in ileal endocrine cells (L cells) triggers the release of glucagon-like peptide 1 (GLP-1) that is transported to the liver through the portal circulation and the pancreas to induce the secretion of insulin by $\beta$ cells to implement its antidiabetic effects such as suppressing appetite and slowing down gastrointestinal (GI) transit [18-20], as well as minimizes the production of proinflammatory cytokines (interleukin (IL)-1, IL-2, IL-6, and tumor necrosis factor, TNF) stimulated by lipopolysaccharides in macrophages and Kupffer cells through the inhibition of nuclear factor kappa B (NF- $\mathrm{kB})$ to assume the responsibility of anti-inflammation, prevention of pathogen invasion, and the maintenance of cell integrity $[18,21,22]$, which also inhibits fibrosis in different tissues such as liver [23]. Primary BA like CA and CDCA are ligands for FXR, while secondary BAs from the microbiota like DCA and LCA are preferential ligands for GPBAR. BAs function as mitogenic and signalling molecules for hepato- 
cytes via FXR and other potential receptors, and can also regulate the status of HSCs. Therefore, drugs that are used to regulate the BAs and to target the specific part of the BAs metabolic process will be potential resources in research and development. It is essential to know the whole metabolic process and regulation for further drug development and therapy.

\section{Characteristics of BAs on Gut}

BAs-associated cytotoxicity, which is direct epithelial toxicity, has been shown to increase the permeability and impair the integrity of the intestinal mucosa, disrupt egg phosphatidylcholine liposomes to facilitate the loss of hydrophobicity and membrane lysis, and induce an increase in IP through changes in tight junction proteins associated with enterocyte anoikis [24]. Glycocholate can decrease ATP production through uncoupling oxidative phosphorylation and increase the ATP-ase activity to induce apoptosis, which results in an increase of permeability across the epithelium by passive diffusion. Exposure of the GI tract to repeated high levels of BAs is a vital risk factor for inflammatory bowel disease (IBD) and GI cancer. BAs are associated with protective effect in intestinal epithelial integrity. BAs also appear to benefit mucosal reconstitution by increased intestinal epithelial cell migration, through an NF- $\kappa \mathrm{B}$ and transforming growth factor beta (TGF- $\beta$ )-mediated pathway to induce more regulatory T-cell downregulating the immune activity [25]. Also, DCA serves as an antimicrobial agent due to its hydrophobicity and detergent characteristics on bacterial membranes; both TGR5 and FXR-dependent signalling mechanisms inhibit gut microbial growth, which play a potential part in guarding against infections from Clostridium difficile and participating in its treatment, particularly aiming at someone in tolerance of antibiotics $[26,27]$. A decrease in total fecal BA concentration, reduction in the ratio of secondary to primary BAs and dysbiosis with overgrowth of Enterobacteriaceae and decrease in autochthonous genera were found in cirrhotic patients compared with controls [6]. Therefore, the transconformation of autochthonous genera to produce secondary BAs to inhibit other bacteria and the metabolites can be used in the metabolism of bacteria and the body. The whole process indicates that the change of gut bacteria may cause the variation of BAs in the gut-liver axis and the toxicity on hepatocytes. However, the bacteria involved in the production of secondary BAs and the result of their loss are still vague and require more experiments.

\section{Regulation from Gut Microbiome}

\section{Perturbation of Microbiome}

Microbiome significantly contribute to the treatment of systemic diseases through signal transduction and activation of the mucosal immune system or via circulating metabolites that reach the hepatic or systemic circulatory system though the portal vein $[28,29]$. Perturbations of the microbiome shape the BA pool and modulate the activity of $\mathrm{BA}$-activated receptors, triggering various metabolic axes and altering host metabolism. Bacterial overgrowth and colonic bacteria move into the proximal parts of the small intestine and disrupt consequently the gut epithelial barrier, facilitating the premature and increased deconjugation of $\mathrm{BA}$ conjugates that are less effective in forming micelles leading to steatorrhea, which can be hindered by the activation of the BA receptor FXR through the regulation of genes, including Ang1, Inos, and IL-18 recognized antimicrobial actions [30]. Some microbial changes are associated with intestinal and systemic inflammation and clinical disease manifestations. A reduction in Firmicutes and Bacteroidetes, and an increase in Proteobacteria including the adherent-invasive Escherichia coli are related to the formation of granulomata and active IBD. Conversely, Faecalibacterium, Roseburia, Lactobacillus, Clostridial clusters and Bacteroides fragilis are associated with reduced intestinal inflammation through the expansion of regulatory $\mathrm{T}$ cells as well as the downregulation of inflammatory cytokine [31].

\section{Enzymes from Specific Bacteria Targeting at BAs to Regulate Metabolism}

Microbial BSHs (bile salt hydrolases) are enzymes catalyzing the hydrolysis process of the $\mathrm{C} 24 \mathrm{~N}$-acyl amide bond of conjugated BAs and are purified from Bacteroides fragilis, Bacteroides vulgatus, Clostridium perfringens, Listeria monocytogenes and several species of Lactobacillus and Bifidobacterium [32, 33], undertake the essential responsibility in gut ecological balance, detoxification feature of which may be involved in some helpful microbial survival in the GI tract in the tolerance of bile salts, while the liberated amino acids from the process such as glycine and taurine may potentially be used as carbon, nitrogen, and energy (mainly in the form of the short chain fatty acids, SCFAs) sources, which could be utilized into bacterial metabolites [34]. SCFA receptor, the free fatty acid receptor 2 that is present on colonic enteroendocrine L cells, has been suggested to serve probably in appetite regulation to enhance the gut hormones 
peptide YY and GLP-1 acutely suppress appetite, contributing to the treatment in obesity. Thus, the production of secondary BAs and the metabolites produced in the process are reasonably anti-metabolic diseases [35]. Fermentation of non-starch polysaccharides by gut microbiota produces an odorous gas composed of various volatile organic compounds, a diverse group of carbon-based volatile chemicals at ambient temperature under conditions of different composition of gut microbiome, exist in the gaseous phase and are present in faeces, urine, exhaled air, and sweat, which reflects microbial metabolic activity; and they are specific biomarkers of colonic as well as metabolic disease. Presumably, the specific indicator of BAs metabolism relative to gut microbiota like one metabolite could be found to be used in the assessment of the stage of liver diseases such as PBC [36]. According to researches and the experience of cross-reactional diseases, some kinds of gut bacteria can express PDC-E2, which share an epitope similar to that of the PDC-E2 of biliary epithelial cells (BECs), resulting in an autoimmune response that causes damage to self cells and tissues. So, gut microbiome play an essential role in $\mathrm{PBC}$ in terms of the regulation of BAs, the expression of cross antigens, and its innate immune response.

\section{Primary Biliary Cirrhosis}

\section{The Pathological Process and Features of PBC}

$\mathrm{PBC}$ is a chronic cholestatic liver disease characterised by the destruction of small intrahepatic bile ducts, and produces a multi-lineage immune response to mitochondrial autoantigens, which could lead to periportal inflammation fibrosis and potential cirrhosis. Therefore, the regulation of the synthesis of BAs and the homeostasis maintenance, as well as the resistance of the BAs toxicity, the regeneration of hepatocytes and the differentiation of mesenchymal stem cells (MSCs) are important and require to be researched further. The main regulatory mechanism of BAs synthesis also involves the gut-liver signalling pathway through FGF19 [14]. PBC is also a model autoimmune disease because of the serological findings (high alkaline phosphatase, ALP), detection of antimitochondrial antibody (AMA), and specific bile duct pathological changes that occur in the disorder, which are sufficient to diagnose the disorder $[37,38]$. The autoimmune nature is characterized by a distinctive loss of tolerance to a series of ubiquitous mitochondrial autoantigens and a strong link between HLA alleles and PBC has been reported [39].
Immune pathways are identified in PBC involving a high concentration of natural-killer T cells, which secrete pro-inflammatory cytokines recorded in patients with PBC [40]. BECs express T-cell ligands, which might act as antigen-presenting cells, amplifying the immune response that is thought to be essential for the induction of autolysis $[41,42]$. Th17 cells abundant in the intestinal lamina propria induced by commensal bacteria and provide protection against invading pathogens, secrete IL-17, IL-21 and TNF- $\alpha$, promoting inflammation and inducing immunosuppressive Treg. These cells participate in epithelial repair by secreting IL-22, which is also proved to be linked to PBC [43]. Hepatocyte injury and non-purulent cholangitis are granulomatous reactions; they involve innate immune responses against BECs determined by the location of the antigen is and by the kind of induced immune response that is created. Also, the gallbladder harbors a complex microbiota, which is presumably similar to the situation in the intestine; the biliary mucosa features a chemical, mechanical, and immunological barrier against commensals. Thus, PBC stimulated by microbial triggers in gallbladder probably induced by imbalance of BAs, may be another cause for the occurrence of the autoimmune liver disease; this aspect require further studies [44]. During cholestasis in the process of $\mathrm{PBC}$, retained BAs modulate immune responses via FXRs in the regulation of liver natural killer T (NKT) cell activation. Also, cholangiocytes are the primary epithelial source of TNF- $\alpha$ in the liver, inducing pro-apoptotic signals through the NF- $\kappa B$ pathway, which is also related to the activation of FXR.

PBC associated with many probable factors like age, gender and overwhelming female predominance [45] manifests clinically with the most common symptoms of fatigue and pruritus, and these symptoms have increased over time [46]. UDCA is the accepted therapy to treat these symptoms, to inhibit intestinal absorption of Bas, to increase biliary secretion of BAs rich in bicarbonate and to eliminate toxic substances from hepatocytes, thereby both enriching the BA pool with less toxic, hydrophilic BAs and relieving the cell necrosis and apoptosis. Besides this, clinical trials of drugs suggest that the administration of obeticholic acid, a 6a-ethyl derivative of CDCA, is well tolerated in patients with $\mathrm{PBC}$ and significantly reduces serum ALP levels [2]. It is definite that BAs assume the dispensable responsibility in PBC. Therefore, the drugs that are used specifically to redress gut dysbiosis and regulate the BAs in homeostasis will be a potential factor influencing the future development of drugs. 
Regeneration and Fibrosis in $P B C$

Regeneration of hepatocyte and fibrosis from activated HSCs is the main pathologic process involved in liver diseases. The flow of bile from the liver to the intestine is obstructed easily, and this is bound to induce the upregulation of CYP7al expression and increase BAs synthesis via negative feedback of FXR-SHP/CYP-FGF19 pathway. This was suggested to ameliorate inflammation maybe by inhibiting TNF- $\alpha$-induced NF- $\kappa \mathrm{B}$ activity and FXR involvement, by the results from the study of WT and TGR5-/- mice and FXR-/- mice, thus illustrating a protecting effect against fibrosis [47]. However, the excessive increase in the production of primary BAs and imbalance in the ratio are cytotoxic and exacerbate hepatocellular injury. Besides, dysbiosis of mirobiome often results from the detriment of a variety of residential bacteria due to the change in composition and quantity of BAs. And during this process, the increase of microbiome products leading to inflammation is absorbed into the liver via portal vein and triggers immune reaction, while there is a decrease in some protective bacteria and their products; however, what they are and how they work are under exploration [48-50].

In PBC, BAs function as mitogenic and signalling molecules for hepatocytes. BAs could initiate hepatocyte proliferation via the activation of FXR. This means that the proper amount of BA retention could induce the regeneration of the intact liver, but the excessive increase of BAs in PBC, which results in cholestatic liver disease, is toxic and apoptotic to aggravate necrosis and apoptosis, thereby leading to fibrosis. So it is reasonable to deem that if the transition period between regeneration and fibrosis, and if the quantity and composition of BAs during this period can be detected, it will be easier to hinder the process via tightly controlling the BAs.

\section{The Regeneration of Hepatocytes and $\alpha$-Catenin}

The liver is renewed to regenerate after injury, and hepatocytes actively divide and establish cell-cell contacts through cell cadherin-catenin complexes. This is the initiative step of intercellular junctions. Classical cadherins are transmembrane glycoproteins that ensure intercellular adhesion through homophilic interactions, and are indirectly connected to the cortical actin cytoskeleton through $\alpha$-catenin, which is a mechanosensor and mechanotransducer with 2 actin-binding domains [51]. The disrupted regenerative capacity is a result of actin cytoskeletal disorganisation, leading to a loss of apical microvilli, dilated and less-contractive lumens in the bile canaliculi, and leaky tight junctions, which indi- cates the essential role of $\alpha$-catenin in liver regeneration. As a molecular switch in the control of actin cytoskeletal organisation during adhesion, ablation of $\alpha$-catenin in the mouse epidermis results in an increase of cell size and proliferation and irregularities in cell polarity [52]. In addition, $\alpha$-catenin knockdown mice exhibited a significant increase in ALP, total bilirubin level, and an accumulation of BAs in the blood circulation, indicating a disruption in bile flow to manifest as a medical condition known as cholestasis in humans with lower high density lipoprotein and signs of inflammation [53]. The manifestations of the ablation of $\alpha$-catenin, partly familiar with the autoimmune-derived cholestasis, may evolve into PBC with the constant existence of defects of adhesion complex and tight junction (fig. 1), which is reasonable to presume that there is a novel and essential relationship between $\alpha$-catenin and PBC. And is there any relationship between BAs and $\alpha$-catenin? Through specific receptors like FXR there could be a relationship between BAs and $\alpha$-catenin but this requires further study.

\section{Activation to Fibrosis and Induced Differentiation of HSC}

Activation to Fibrosis

The liver is a central immunological organ with a high exposure to circulating antigens and endotoxins from the gut microbiota, enriched with innate immune cells that show its tolerance of wide scopes, while it can also rapidly activate immunity in response to different triggers like infections and tissue damage. It is well known that stellate cells store retinoids mainly as retinyl palmitate at the quiescent state of HSC and are lost when cells are activated to develop into proliferating myofibroblast-like cells to produce extracellular matrix proteins to partly contribute to fibrogenesis and scar formation in chronic liver diseases. The inflammatory activation of hepatic stellate and Kupffer cells results in the chemokine-mediated infiltration of neutrophils, monocytes, natural killer and NKT cells, during which the hepatocytes are injured and undergo fibrosis to resist inflammation, but result in the aberrant ECM synthesis and remodeling, which lead to tissue stiffening and loss of organ function [54]. As an important component of the liver innate immune system, liver sinusoidal endothelial cells (LSECs) with fenestrations, provide a porous barrier that facilitates the access of hepatocytes to oxygen and small molecules in the microcirculation, and clear colloids and macromolecules, which promotes HSCs quiescence and induces immune tolerance [55]. However, capillarization or defenestration 


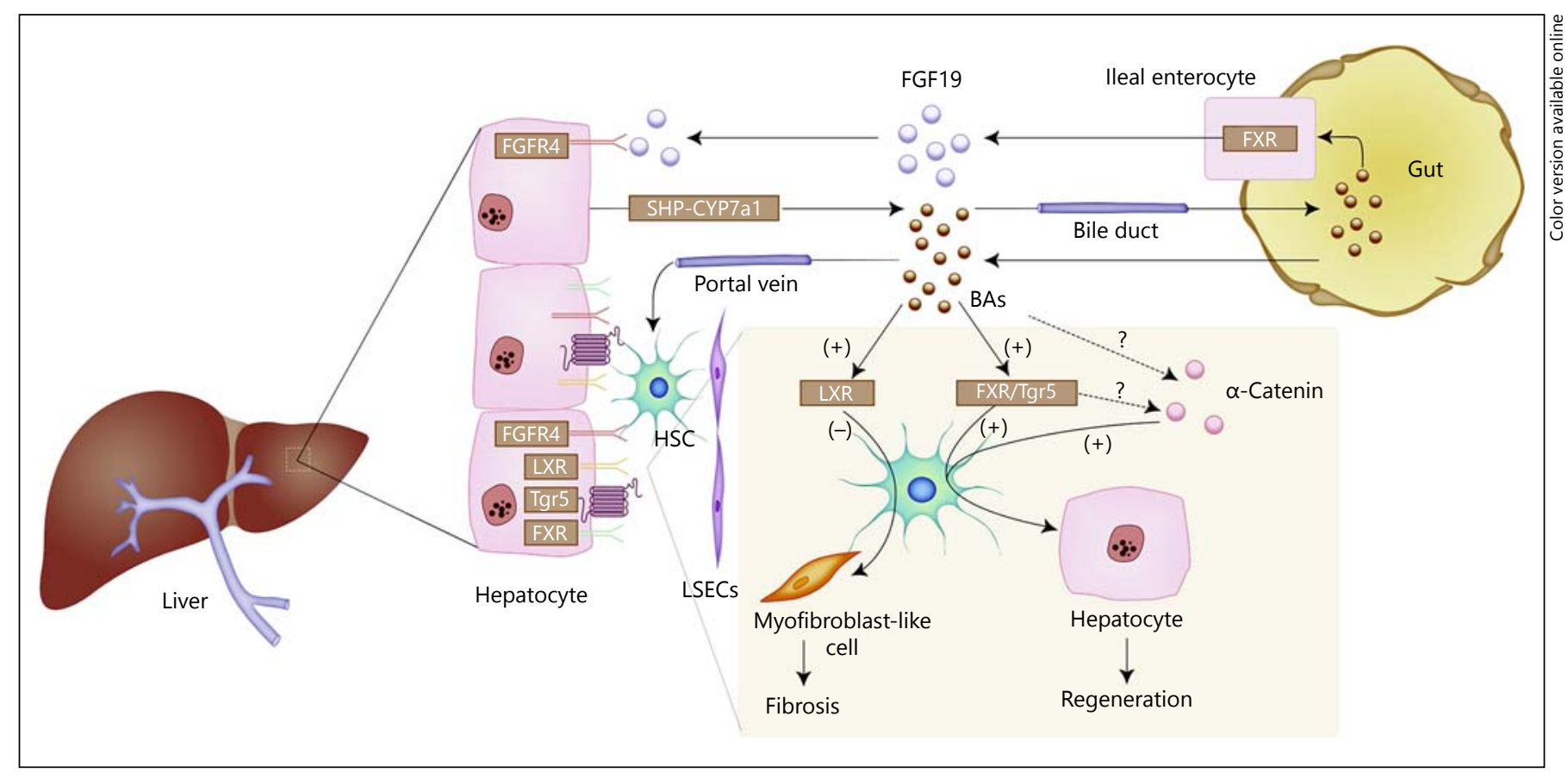

Fig. 1. The composition and proportion of BAs are highly influenced by gut microbiota, which indicates that the ecosystem of gut bacteria assumes an essential responsibility in autoimmune liver diseases relevant with BAs. Recent researches suggest that BAs trigger the differentiation of the HSCs into hepatocyte-like cells in vitro to facilitate liver regeneration, which involves the BA receptors Fxr and Tgr5. Also, BAs can active the LXR to hinder the
LSECs capillarization to tranquilize the HSCs and then reduce the production of ECM to inhibit the fibrosis. The $\alpha$-catenin is activated to participate in the regeneration of hepatocytes. So it is reasonable to speculate that there is probable direct connection between BAs and $\alpha$-catenin, or indirectly through FXR or Tgr5, which promote the liver regeneration in cooperation with the 2 pathways mentioned above during the early phase of the PBC. of LSECs triggered by the formation and deposition of extracellular matrix in the space of Disse, results in LSECs dysfunction, and occurs prior to the activation of the HSCs and the development of fibrosis. Restoration of LSECs leads to the HSCs quiescence and the regression of fibrosis. Nuclear receptors: LXRs (LXR $\alpha$ and LXR $\beta$ ) influence the activation of HSCs and contribute to the pathogenesis of liver fibrosis. They are also the receptors of BAs, probably identified as a novel target for antifibrotic therapy. The activation of LXRa can inhibits LSECs capillarization and HSCs activation, and resists CCl4-induced liver fibrosis [56], which indicates that the imbalance of BAs may be the key reason leading to capillarization or defenestration of LSECs (fig. 1). Furthermore, HSCs are the primary source of myofibroblasts in liver fibrosis. Their activation is also associated with the magnitude and the timing of mechanical signals in mechanotransduction signalling dynamics, and they promote the defenestration of LSECs. Some experiments showed that HSCs seeded on soft substrates remained quiescence with lipid droplets, whereas stellate cells cultured on stiff sub- strates remained activated while losing their lipid droplets. Meanwhile, stellate cells initially cultured on soft gels, subjected to secondary cross-linking responded rapidly to the change in substrate stiffness, resembling the myofibroblast-like morphology, which indicates that there is a positive feedback between fibrosis and LSECs capillarization [57].

\section{Differentiation of HSC Induced by BAs}

Recent researches suggest that the differentiation of MSCs is essential, and their therapeutic potential has become obvious in differentiating into multiple cell lineages, which may serve a potential role in PBC therapy. HSCs are the most typical kind of MSCs evidenced by their MSC-related expression pattern and functional analyses; they potentially differentiate into epithelial cell lineages such as hepatocytes and cholangiocytes derived from the bone marrow or adipose tissue after the stimulation of BAs to promote liver regeneration [58]. Physiological concentrations of BAs such as tauroursodeoxycholic acid (TUDCA) were able to initiate hepatic differentia- 
tion of MSC via the FXR and Tgr5 as investigated, which makes the clinical therapy a very important forward step ahead [59-63]. BAs as mentioned before are recognized as important regulators of metabolism, also as mediators of liver regeneration, since the concentration of many BAs in the blood serum of rats transiently increased after liver injury by partial hepatectomy, in which TUDCA was found to mediate the induction hepatic differentiation at the concentration of $2 \mu \mathrm{M}$ among the BAs tested [64]. Thus, physiological concentrations of BAs can trigger the differentiation of rodent and human MSC into hepatocyte-like cells in vitro, which involves the BA receptors Fxr and Tgr5 as well as several signalling pathways such as the notch, hedgehog and Tgf- $\beta / \mathrm{Bmp}$ signalling pathway (fig. 1).

The TUDCA-induced hepatic differentiation of activated bmMSC was remarkably inhibited by the knockout of either Fxr or Tgr5 [61]. TUDCA was found to be the most potent among the BAs tested but a weak ligand of Fxr and Tgr5, suggesting that other essential pathways via BAs are probably involved to mediate this process. As mentioned above, BAs can activate the LXR to hinder the LSECs capillarization to tranquilize the HSCs and then reduce the production of ECM to inhibit the fibrosis. Meanwhile, UDCA increases biliary secretion of BAs and the elimination of toxic substances from hepatocytes, and relieves the cell necrosis and apoptosis induced by BA accumulation; and during the whole process, the $\alpha$-catenin was activated to participate in the regeneration of hepatocytes. So it is reasonable to speculate that there is probably a direct connection between BAs and $\alpha$-catenin, or an indirect link through FXR or TGR5, which promotes cooperative regeneration. Other questions are as follows: Will a slight increase in BAs induce liver regeneration? Will a severe increase in BAs result in necrosis and fibrosis? Will it be crucial what the transition phase of the quality of BAs is and will there be any indicator to facilitate its detection? Will the LXR be downregulated in high concentrations of BAs? Answers to all these questions are to be revealed only through further experiments.

\section{Conclusion}

PBC is a chronic cholestatic liver disease and also a model autoimmune disease with AMA (PDC-E2), and UDCA is the only accepted therapy for a kind of BAs. The liver is a central immunological organ with a high exposure to circulating antigens, which share a similar epitope as of PDC-E2 of BECs and endotoxins from the gut mi- crobiota to result in immune response. BAs function as mitogenic and signalling molecules for hepatocytes via receptors mentioned, and are also involved in the induction of HSCs conversation, which assumes a potential role in facilitating liver regeneration and anti-fibrosis. However, the activation of HSC after liver injury resists inflammation but causes fibrosis. The transitional period between regeneration and fibrosis may exist on the basis of BAs' multiple functions. Also, the process of differentiating induction and activation of HSC has its own signalling pathway and triggers, but the requirements such as specific triggers or inhibitors needed for changing over fibrosis to differentiation in order to promote the autoregeneration in the early phase of $\mathrm{PBC}$ and not allow the onset of activation are still unknown and require more study and exploration. Both BAs and gut microbiome, especially bacteria with $7 a$-dehydroxylase, are abnormal in PBC; this shows the complex interaction between metabolism and composition of BAs and the composition of gut microbiome; this finding requires to be validated with more research.

\section{Confirmation and Statements}

1. Y.H. and Z.D. have contributed to and agreed on the content of the manuscript.

2. The manuscript has not been published previously, in any language, in whole or in part, and is not currently under consideration elsewhere.

3. There are no ethical problems.

4. There is no conflict of interest in relation to any funding from or pecuniary interests in companies that could be perceived as a potential conflict of interest in the outcome of the research.

\section{References}

$$
\begin{aligned}
& 1 \text { Watanabe M, Houten SM, Wang L, et al: Bile } \\
& \text { acids lower triglyceride levels via a pathway } \\
& \text { involving FXR, SHP, and SREBP-1c. J Clin In- } \\
& \text { vest 2004;113:1408-1418. } \\
& 2 \text { Mousa HS, Lleo A, Invernizzi P, et al: Advanc- } \\
& \text { es in pharmacotherapy for primary biliary cir- } \\
& \text { rhosis. Expert Opin Pharmacother 2015; } 16 \text { : } \\
& \text { 633-643. } \\
& 3 \text { Salonen A de Vos WM: Impact of diet on hu- } \\
& \text { man intestinal microbiota and health. Annu } \\
& \text { Rev Food Sci Technol 2014;5:239-262. } \\
& 4 \text { Vavassori P, Mencarelli A, Renga B, et al: The } \\
& \text { bile acid receptor FXR is a modulator of intes- } \\
& \text { tinal innate immunity. J Immunol 2009; } 183 \text { : } \\
& \text { 6251-6261. } \\
& 5 \text { Cipriani S, Mencarelli A, Chini MG, et al: The } \\
& \text { bile acid receptor GPBAR-1 (TGR5) modu- } \\
& \text { lates integrity of intestinal barrier and im- } \\
& \text { mune response to experimental colitis. PLoS } \\
& \text { One 2011;6:e25637. }
\end{aligned}
$$


6 Kakiyama G, Pandak WM, Gillevet PM, et al: Modulation of the fecal bile acid profile by gut microbiota in cirrhosis. J Hepatol 2013;58: 949-955.

7 Vitek L: Bile acid malabsorption in inflammatory bowel disease. Inflamm Bowel Dis 2015; 21:476-483.

8 Modica S, Gadaleta RM, Moschetta A: Deciphering the nuclear bile acid receptor FXR paradigm. Nucl Recept Signal 2010;8:e005.

9 Hofmann AF, Hagey LR: Key discoveries in bile acid chemistry and biology and their clinical applications: history of the last eight decades. J Lipid Res 2014;55:1553-1595.

10 Hofmann AF: Detoxification of lithocholic acid, a toxic bile acid: relevance to drug hepatotoxicity. Drug Metab Rev 2004;36:703-722.

11 Fiorucci S, Distrutti E: Bile acid-activated receptors, intestinal microbiota, and the treatment of metabolic disorders. Trends Mol Med 2015;21:702-714.

12 Watanabe M, Horai Y, Houten SM, et al: Lowering bile acid pool size with a synthetic farnesoid X receptor (FXR) agonist induces obesity and diabetes through reduced energy expenditure. J Biol Chem 2011;286:2691326920.

13 Tsuei J, Chau T, Mills D, et al: Bile acid dysregulation, gut dysbiosis, and gastrointestinal cancer. Exp Biol Med (Maywood) 2014;239: 1489-1504.

14 Wunsch E, Milkiewicz M, Wasik U, et al: Expression of hepatic Fibroblast Growth Factor 19 is enhanced in Primary Biliary Cirrhosis and correlates with severity of the disease. Sci Rep 2015;5:13462.

15 Kullak-Ublick GA, Stieger B, Meier PJ: Enterohepatic bile salt transporters in normal physiology and liver disease. Gastroenterology 2004;126:322-342.

16 Zhou X, Cao L, Jiang C, et al: PPARa-UGT axisactivation repressesintestinalFXR-FGF15 feedback signalling and exacerbates experimental colitis. Nat Commun 2014;5:4573.

17 Potthoff MJ, Boney-Montoya J, Choi M, et al FGF15/19 regulates hepatic glucose metabolism by inhibiting the CREB-PGC-1 $\alpha$ pathway. Cell Metab 2011;13:729-738.

18 Keitel V, Donner M, Winandy S, et al: Expression and function of the bile acid receptor TGR5 in Kupffer cells. Biochem Biophys Res Commun 2008;372:78-84.

19 Katsuma S, Hirasawa A, Tsujimoto G: Bile acids promote glucagon-like peptide-1 secretion through TGR5 in a murine enteroendocrine cell line STC-1. Biochem Biophys Res Commun 2005;329:386-390.

20 Inagaki T, Choi M, Moschetta A, et al: Fibroblast growth factor 15 functions as an enterohepatic signal to regulate bile acid homeostasis. Cell Metab 2005;2:217-225.

21 Smith PM, Howitt MR, Panikov N, et al: The microbial metabolites, short-chain fatty acids, regulate colonic Treg cell homeostasis. Science 2013;341:569-573.

22 Furusawa Y, Obata Y, Fukuda S, et al: Commensal microbe-derived butyrate induces the differentiation of colonic regulatory $\mathrm{T}$ cells. Nature 2013;504:446-450.

23 Lua I, Asahina K: The role of mesothelial cells in liver development, injury, and regeneration. Gut Liver 2016;10:166-176.

24 Raimondi F, Santoro P, Barone MV, et al: Bile acids modulate tight junction structure and barrier function of Caco-2 monolayers via EGFR activation. Am J Physiol Gastrointest Liver Physiol 2008;294:G906-G913.

25 Strauch ED, Yamaguchi J, Bass BL, et al: Bile salts regulate intestinal epithelial cell migration by nuclear factor-kappa B-induced expression of transforming growth factor-beta. J Am Coll Surg 2003;197:974-984.

26 Sayin SI, Wahlström A, Felin J, et al: Gut microbiota regulates bile acid metabolism by reducing the levels of tauro-beta-muricholic acid, a naturally occurring FXR antagonist. Cell Metab 2013;17:225-235.

27 Buffie CG, Bucci V, Stein RR, et al: Precision microbiome reconstitution restores bile acid mediated resistance to Clostridium difficile. Nature 2015;517:205-208.

28 Wang L, Lee YK, Bundman D, et al: Redundant pathways for negative feedback regulation of bile acid production. Dev Cell 2002;2: 721-731.

29 Wikoff WR, Anfora AT, Liu J, et al: Metabolomics analysis reveals large effects of gut microflora on mammalian blood metabolites. Proc Natl Acad Sci U S A 2009;106:36983703.

30 Inagaki T, Moschetta A, Lee YK, et al: Regulation of antibacterial defense in the small intestine by the nuclear bile acid receptor. Proc Natl Acad Sci U S A 2006;103:3920-3925.

31 Duboc H, Rainteau D, Rajca S, et al: Increase in fecal primary bile acids and dysbiosis in patients with diarrhea-predominant irritable bowel syndrome. Neurogastroenterol Motil 2012;24:513-520, e246-e247.

32 Begley M, Gahan CG, Hill C: The interaction between bacteria and bile. FEMS Microbiol Rev 2005;29:625-651.

33 Jones BV, Begley M, Hill C, et al: Functional and comparative metagenomic analysis of bile salt hydrolase activity in the human gut microbiome. Proc Natl Acad Sci U S A 2008; 105:13580-13585.

34 Theriot CM, Young VB: Interactions between the gastrointestinal microbiome and clostridium difficile. Annu Rev Microbiol 2015;69: 445-461.

35 Psichas A, Sleeth ML, Murphy KG, et al: The short chain fatty acid propionate stimulates GLP-1 and PYY secretion via free fatty acid receptor 2 in rodents. Int J Obes (Lond) 2015; 39:424-429.

36 Arasaradnam RP, Covington JA, Harmston C, et al: Review article: next generation diagnostic modalities in gastroenterology-gas phase volatile compound biomarker detection. Aliment Pharmacol Ther 2014;39:780-789.

37 Lindor KD, Gershwin ME, Poupon R, et al: Primary biliary cirrhosis. Hepatology 2009; 50:291-308.
38 Nakamura M: Clinical significance of autoantibodies in primary biliary cirrhosis. Semin Liver Dis 2014;34:334-340.

39 Invernizzi P, Selmi C, Poli F, et al: Human leukocyte antigen polymorphisms in Italian primary biliary cirrhosis: a multicenter study of 664 patients and 1992 healthy controls. Hepatology 2008;48:1906-1912.

40 Carbone M, Lleo A, Sandford RN, et al: Implications of genome-wide association studies in novel therapeutics in primary biliary cirrhosis. Eur J Immunol 2014;44:945-954.

41 Selmi C, Lleo A, Pasini S, et al: Innate immunity and primary biliary cirrhosis. Curr Mol Med 2009;9:45-51.

42 Henao-Mejia J, Elinav E, Thaiss CA, et al: Role of the intestinal microbiome in liver disease. J Autoimmun 2013;46:66-73.

43 Trivedi PJ, Adams DH: Mucosal immunity in liver autoimmunity: a comprehensive review. J Autoimmun 2013;46:97-111.

44 Verdier J, Luedde T, Sellge G: Biliary mucosal barrier and microbiome. Viszeralmedizin 2015;31:156-161.

45 Talwalkar JA, Lindor KD: Primary biliary cirrhosis. Lancet 2003;362:53-61.

46 Boonstra K, Kunst AE, Stadhouders PH, et al: Rising incidence and prevalence of primary biliary cirrhosis: a large population-based study. Liver Int 2014;34:e31-e38.

47 Liu H, Pathak P, Boehme S, Chiang JY: Cholesterol 7a-hydroxylase protects the liver from inflammation and fibrosis by maintaining cholesterol homeostasis. J Lipid Res 2016; 57:1831-1844.

48 Verdier J, Luedde T, Sellge G: Biliary mucosal barrier and microbiome. Viszeralmedizin 2015;31:156-161.

49 Kakiyama G, Pandak WM, Gillevet PM, Hylemon PB, Heuman DM, Daita K, Takei $\mathrm{H}$, Muto A, Nittono H, Ridlon JM, White MB, Noble NA, Monteith P, Fuchs M, Thacker LR, Sikaroodi M, Bajaj JS: Modulation of the fecal bile acid profile by gut microbiota in cirrhosis. J Hepatol 2013;58:949-955.

50 Hofmann AF, Eckmann L: How bile acids confer gut mucosal protection against bacteria. Proc Natl Acad Sci U S A 2006;103:4333-4334.

51 Hansen SD, Kwiatkowski AV, Ouyang CY, et al: $\alpha$ E-catenin actin-binding domain alters actin filament conformation and regulates binding of nucleation and disassembly factors. Mol Biol Cell 2013;24:3710-3720.

52 Schlegelmilch K, Mohseni M, Kirak O, et al: Yap1 acts downstream of $\alpha$-catenin to control epidermal proliferation. Cell 2011;144:782795.

53 Herr KJ, Tsang YH, Ong JW, et al: Loss of a-catenin elicits a cholestatic response and impairs liver regeneration. Sci Rep 2014;4: 6835.

54 Friedman SL: Hepatic stellate cells: protean, multifunctional, and enigmatic cells of the liver. Physiol Rev 2008;88:125-172.

55 Racanelli V, Rehermann B: The liver as an immunological organ. Hepatology 2006;43(2 suppl 1):S54-S62. 
56 Xing Y, Zhao T, Gao X, et al: Liver X receptor $a$ is essential for the capillarization of liver sinusoidal endothelial cells in liver injury. Sci Rep 2016;6:21309.

57 Caliari SR, Perepelyuk M, Cosgrove BD, et al: Stiffening hydrogels for investigating the dynamics of hepatic stellate cell mechanotransduction during myofibroblast activation. Sci Rep 2016;6:21387.

58 Geerts A: On the origin of stellate cells: mesodermal, endodermal or neuro-ectodermal? J Hepatol 2004;40:331-334.
59 Kordes C, Sawitza I, Götze S, et al: Hepatic stellate cells support hematopoiesis and are liver-resident mesenchymal stem cells. Cell Physiol Biochem 2013;31:290-304.

60 Castilho-Fernandes A, de Almeida DC, Fontes AM, et al: Human hepatic stellate cell line (LX-2) exhibits characteristics of bone marrow-derived mesenchymal stem cells. Exp Mol Pathol 2011;91:664-672.

61 Kordes C, Sawitza I, Götze S, et al: Hepatic stellate cells contribute to progenitor cells and liver regeneration. J Clin Invest 2014;124: 5503-5515.
62 Swiderska-Syn M, Syn WK, Xie G, et al: Myofibroblastic cells function as progenitors to regenerate murine livers after partial hepatectomy. Gut 2014;63:1333-1344.

63 Kordes C, Sawitza I, Müller-Marbach A, et al: CD133+ hepatic stellate cells are progenitor cells. Biochem Biophys Res Commun 2007; 352:410-417.

64 Sawitza I, Kordes C, Götze S, et al: Bile acids induce hepatic differentiation of mesenchymal stem cells. Sci Rep 2015;5:13320. 